Review

\title{
TECHNICAL PARAMETERS AND CRITERIA FOR VIRTUAL BRONCHOSCOPY IN DIAGNOSTICS OF TRACHIAL AND BRONCHIAL RUPTURES
}

\author{
M. Mitev* \\ Department of Medical Physics, Biophysics, Roentgenology and Radiology, Faculty of Medicine, \\ Department of Diagnostic Imaging, University Hospital 'St. Kirkovich', Trakia University - \\ Stara Zagora, Bulgaria
}

\begin{abstract}
VB is a non-invasive technique and follow-up tool tolerant of most patients, requiring a precise method of diagnosis, especially in ruptures with the formation of high-grade stenoses for which FB is not applicable, as well as in children and elderly or severely ill patients. The study aims to track the technical parameters, key indicators, and criteria for optimizing the diagnostic capabilities of Virtual bronchoscopy (VB) in tracheal and bronchial ruptures. Optimization of the technical parameters of the scans taking into account the age of the patient, the weight, the size, the nature of the ruptures and the severity of the diagnosis; the application of appropriate criteria and parameters for the diagnosis and evaluation of the tracheobronchial tree, combined with the capabilities of modern equipment, guarantee the high reliability, sensitivity, and specificity of the VB method.
\end{abstract}

Key words: ruptures, trachea, bronchi, Virtual bronchoscopy, technical parameters,

\section{INTRODUCTION}

Virtual Bronchoscopy (VB) is a postprocessing technique that presents $3 \mathrm{D}$ images of the inner surface of the tracheobronchial tree simulating endoluminal views. In combination with axial and multiplanar reconstruction (MPR) of MDCT images, VB can increase diagnostic accuracy in a number of tracheobronchial endoluminal pathologies (1). Multidetector computed tomography (MDCT) plays a key role in identifying and classifying a large number of respiratory diseases and, together with post-treatment tools such as VB, can improve the effectiveness of the study (2). There are relatively few studies in the scientific literature presenting detailed data on the technical parameters, basic criteria and indicators for the successful application of the VB method in the diagnosis of tracheal and bronchial ruptures (3-4, 2, 5, etc.). The present work aims to follow the experience of

Correspondence to: D-r Mitko Atanasov Mitev, DM, Department of Medical Physics, Biophysics,

Roentgenology and Radiology, Faculty of Medicine,

Department of Diagnostic Imaging, University Hospital

"St. Kirkovich", Trakia University - Stara Zagora, 6000

Stara Zagora, Bulgaria, Mobile: +359887 706079, E-

mail: mitev.mitko69@gmail.com researchers in improving the accuracy, success of VB in the diagnosis of tracheal and bronchial ruptures.

\section{RESULTS AND DISCUSSION}

Optimization of the technical parameters, the main criteria and indicators are of crucial importance for the successful application of the VB method in the diagnosis of tracheal and bronchial ruptures. Jones and Athanasiou (3) compare the precision of the FB ("gold standard") and VB methods as tools for the diagnosis, grading and monitoring of pulmonary diseases. The indicators determine the specificity and sensitivity of the two methods based on the results of diagnostic studies in 459 patients. For true-positive findings, these anomalies are found, which are found by both methods. Those who are defined as normal by both methods (true-negative findings) are considered as real negative cases. False-positive findings determine when reported as abnormalities after VB and normal after FB. False-negative findings are defined as those diagnosed with inappropriate diagnosis after VB and as anomalies after FB. They examine the lesions and the degree of stenosis. They point out that low collimation is 
associated with better image quality. Studies using a maximum of $3 \mathrm{~mm}$ collimation were analyzed with better accuracy. Statistical analyses were performed using the SPSS software package version 11.0 for Windows (SPSS Inc, Chicago, IL) and the meta-test software version 0.9 (developed by Joseph Lau). For the examination of tracheobronchial lesions and for suspected respiratory tract lesions use the technical parameters quoted by Jones and Athanasiou (3): Pitch - 1.5, $150 \mathrm{~mA}$ current in the tube, collimation $2 \mathrm{~mm}$, voltage in the tube $120 \mathrm{~V}$, reconstruction interval 1 mm (by the studies of Matute et al. (6); 170 $\mathrm{mA}$ current in the pipe, collimation 1-3 mm, a voltage in the pipe $120-130 \mathrm{~V}$, reconstruction interval 1-2 $\mathrm{mm}$ and threshold -500 in Hansfield units (according to research by Schoepf et al., (7). Following VB at the indicated technical parameters, Jones and Athanasiou (3) reported an average specificity of 94\% (86\% -97\%); sensitivity 94\% (86\% $97 \%$ ), while those after FB are average specificity $84 \%(76 \%-90 \%)$; sensitivity $72 \%$ $(58 \%-84 \%))$. These results, according to the authors, warrant VB's determination with excellent precision in diagnosing patients with suspected respiratory tract lesions. Maniatis et al. (8) examined the potential of VB for the diagnosis of bronchial abnormalities with moderate and low stenosis in 65 patients (54 men and 11 women; age 22-78 years; mean age $64.5 \pm 3$ years) over two years. In 30 of the studied patients, after FB revealed bronchial abnormalities, incl. Endobronchial lesions and stenosis $(75 \%)$. All patients then underwent a spiral CT scan between $3.1 \pm 3$ days. Patients are informed of the consent of the patients after they have been informed in writing, following guidelines laid down by the General Hospital" Konstantopoulio Agia Olga" Hospital, Athens, Greece. FB is used as the standard for determining positive and negative cases. They use an Olympus P20D bronchoscope (Olympus America, Inc., Melville, New York, USA) connected to an external monitor. Analyze the indicators: type of bronchial anomaly, location, a degree of stenosis. The degree of bronchial stenosis is subjectively defined in the categories: grade 1 (stenosis of $0-25 \%$ ), grade 2 (stenosis of $25-50 \%$ ), grade 3 (stenosis of 50-75\%), grade 4 (stenosis of 7599\%) and grade 5 (100\% stenosis). Spiral CT was performed with a CT scanner (Prospeed SX Power; G.E. Medical Systems, Milwaukee, Wisc., USA) with the following technical parameters: $120 \mathrm{kV}, 160 \mathrm{mAs}, 3 \mathrm{~mm}$ collimation, pitch 1.5 and a $512 \times 512$ matrix.
These parameters are determined after selecting the optimal scan parameters for VB $(9-10,8)$. The scan time used was $28-34$ s for a period of one deep breath after hyperventilation. Patients scan in a caudiocranial direction to minimize movement. To reconstruct the scanned images to $3 \mathrm{~mm}$, they use a $1 \mathrm{~mm}$ reconstruction interval and a standard virtual simulation algorithm to increase the spatial resolution along the z-axis. The resulting series of images are transferred over a high-speed internet network to a workstation (Sun Sparcstation 20; Sun Microsystems, Mountain View, Calif., USA) with the software Advantage Windows (G.E. Medical Systems) installed. The bronchial tree is divided into ten sections (trachea-right-left main bronchus, right upper-intermediatemiddle-lower lobe bronchus, left upper-lingula, and lower lobe bronchus). If the lesion covers more than one bronchial section, they are evaluated individually. All stenotic processes, unevenness of the bronchial wall, displacement of the bronchi, and intraluminal lesions $(11,8)$ have been observed for bronchial abnormalities. The thin-layer CT image reconstruction is performed directly on a 21 inch workstation monitor. The entire series of images are loaded into the browser included with the Advantage Windows software and viewed without MPR reconstruction or other three-dimensional imaging. The resulting thinlayer CT images were processed on the same workstation with Navigator 2.0.6 (G.E. Medical Systems) virtual endoscopy software, allowing 4 on-screen images: virtual intraluminal image; primary axial image; reformatted coronal image; reformatted sagittal image. The software used provides the 'flythrough sequence' tool for navigating the bronchial tree. For the reconstruction of the virtual bronchial wall, the threshold values from 2520 to $2720 \mathrm{HU}$ are used. They operate at a corrected angle of the virtual endoscope cone in the range of $30^{\circ}$ to $55^{\circ}$, depending on the location and type of bronchial anomaly. Statistical processing of data is performed with the help of MedCalc 7.2.0.2 software package (MedCalc Software, Mariakerke, Belgium) with non-parametric data analysis methods and the Windows XP operating system $(12,8)$. Maniatis et al. (8) found that VB provided a more accurate estimate of thin-film CT, as well as no statistically significant differences with FB results, as opposed to CT. The technical parameters and apparatus thus used achieve over $97 \%$ specificity and sensitivity of VB in the range of $90-94 \%$. Anthracopoulos et al. 
(13) present VB and other 3D Imaging methods for the diagnosis of the tracheobronchial tree. According to them, Flexible bronchoscopy (FB) is the method of real-time direct visualization and dynamic evaluation of the tracheobronchial system, and Multidetector computed tomography (MDCT) generates accurate $2 \mathrm{D}$ and $3 \mathrm{D}$ images (including VB) of the airways. The authors highlight the advantages of new imaging techniques compared to those of FB: accurate mapping of airway compression or stenosis, visualization of the airways beyond the obstruction area, assessment of smaller airways, and imaging of parenchymal and mediastinal abnormalities. They state that the ALARA principle of 'as low as reasonably achievable' should be applied concerning the problem of patient exposure. They confirm the undisputed place of FB as the "gold standard" for the diagnosis of respiratory tract abnormalities but point out that it is an invasive procedure and cannot be used in clinical practice for high-grade bronchial lumen stenoses. FB, according to the authors, is often combined with MDCT, and the advent of powerful scanners (64-, 128-, 256-, and even 320-slice scanners) makes it possible to get images in seconds without applying the breath-hold method. They point out that MDCT provides continuous and complete sets of raw data that are transferred to an image archiving system and communication system or three-dimensional post-processing and analysis workstation, and once the final data set is received, apply different algorithms to generate accurate $2 \mathrm{D}$ and $3 \mathrm{D}$ images based on scan information. They present VB as a noninvasive technique and tracking tool, providing images and on-line videos well tolerated by most patients for whom MDCT is the main source of data. The authors point out that through submillimeter collimation at MDCT, technology can achieve deeper penetration, allowing VB to accurately map the airways from 6 to 7 lines in adults and from 3 to 4 lines in children. The authors' experience has shown that MDCT imaging in children should be performed using standardized low dose protocols, consistent with weight and diagnostic issues. The authors state that highquality 3D images are needed in children: scanning speed $(\leq 1 \quad \mathrm{~s})$, lower collimation (0.625-0.75 $\mathrm{mm}$ for 16-row and 0.5-0.6 mm for a 64-row detector with a pitch of 1.0-1.5), and to improve the 3D image for slices over 1 $\mathrm{mm}$ thick, reconstruction with overlays of slides of about $50 \%$ is recommended, etc.
Mirka et al. (4) present MDCT as the most important method for imaging_chest trauma. MDCT's advantage is the high speed and high geometric resolution of the method in each plane. The method allows large airways to be imaged and precision, multiplanar 3D images created, making significantly more accurate diagnoses. This motivates, according to the authors, the choice of MDCT in severe trauma. The authors note that the clinical symptoms of breast injuries are varied and often do not correlate with their severity, which is why the diagnostic imaging is first started as soon as the patient is admitted. MDCT allows 2D and 3D reformations to be performed in any plane without loss of geometric resolution. The authors emphasize that diagnosis requires a high rate of research, and the need for reformation in other planes requires high resolution on the z-axis. They recommend the use of appropriate exposure parameters, which could be reduced by 5-10 times in children compared to adults. According to them, this is achieved not only by automatic dose modulation but also by adjusting the reference exposure values according to body weight. The authors point out that the value of kilovolts can be reduced to $80-100$ and the value of mAs can be reduced to $30-80$ for children. For adults, the technical specifications for scanning are: voltage $120 \mathrm{kV}$; current $80-120 \mathrm{mAs}$; rotation time $0.27-0.5 \mathrm{~s}$; collimation $0.6 \mathrm{~mm}$; pitch 0.9-1.5; scan duration time 1-3 sec. They conclude that MDCT is a major component of the diagnostic algorithm for chest injuries. Lobo and Antunes (14) published studies on the use of CT of the thorax in infants and children, focusing mainly on significant technical aspects and clinical applications in the evaluation of the lung, mediastinum, and chest wall. According to Barnes et al. (2), FB visualizes the mucosal layer and is an important method for therapy and diagnosis. A significant drawback of the method is the inability to use it to evaluate deep layers or adjacent tissues. The authors indicate that MDCT and after treatment techniques, such as $\mathrm{VB}$, provide an excellent evaluation of the airway wall. MDCT requires $1 \mathrm{~mm}$ axial thin sections and $80 \%$ overlap defined as optimal parameters, multiplanar reformations, minimum intensity projections, volumerendered images, VB images and more. They also stress the importance of an adequate interpretation of the results obtained. Mitev et al. (15) investigate the diagnostic capabilities of VB in the study of tracheal and bronchial ruptures from advanced oesophagal neoplasm. 
VB research is done with 64 MDCTs of 'Siemens Definition AS'. The scanning is performed with air retention for 10 seconds, adjusted automatically with contrast or native. Subsequent processing of $0.6 \mathrm{~mm}$ MDVB scratches based on the reconstructed images obtained. The additional processing is performed with the VB protocol. They use the Siemens workstation with Singovia VB10 software and the Siemens Definition AS workstation with tracking and Axial, coronal and sagittal images comparison. Multiplanar reconstructions are performed using MIP techniques and backup options and exporting images and video. Optimize standard protocols for patients with different diagnoses and age groups, using different slice thickness, different amperage, voltage and pitch. The diagnostic capabilities of VB versus FB are compared based on the following criteria: location of the rupture; the shape of the rupture; length of rupture $(\mathrm{mm})$; damaging the integrity of the tracheal rings; a distance of the rupture to the customs; a distance of the rupture to the rhyme and the vocal cords. The authors defined VB with MDCT as a basic and expert diagnostic method, applicable and recommended in cases with damage to the respiratory tract where fiberoptic bronchoscopy may not be performed, e.g., in cases of a high degree of stenoses. Kotlyarov (5) performed Multispiral computed tomography (MSCT) on 128-slice computed tomography of the company GE Healthcare, model "Optima CT 660" and post-processing data processing, obtaining virtual bronchograms, and 3D imaging - at the workstation "Optima CT 660". 3D images are obtained from Advantage Workstation (GE), Toshiba Aquilion 16 (16-slice) and Aquilion ONE (320-slice) workstations. For the reconstruction of 3D data in virtual bronchoscopy images, the author uses a threedimensional modelling technique that produces a three-dimensional array with images of the inner and outer surfaces of the bronchi, based on which VB examines the tracheobronchial tree using the VB fly-through method and extensive reconstruction of the lung and its structures.

\section{CONCLUSION}

Achieving high precision of the VB method, high specificity and sensitivity of the method requires: modern suitable equipment and software products adapted to it; highly qualified specialists who can correctly interpret the results obtained; optimization of work protocols according to the severity and nature of the ruptures obtained, the age of the patients examined, the weight, size, concomitant diseases; speed of conducting, etc. VB is blurred as a tool of high precision diagnostics, especially in ruptures with the formation of high-grade stenoses for which FB is not applicable, as well as in children and elderly or severely ill patients.

\section{REFERENCES}

1. Debnath J., George R.A., Satija L., Ahmed S., Rai S. P., Roy S., Virtual bronchoscopy in the era of multi-detector computed tomography: Is there any reality? Med $J$ Armed Forces India, 69(3):305-310, 2013. doi:10.1016/j.mjafi.2012.12.005

2. Barnes, D., Chacoff, J. G., Benegas, M., Perea, R. J., de Caralt, T. M., Ramirez, J., Vollmer, I. Central airway pathology: clinic features, CT findings with pathologic and virtual endoscopy correlation. Insights Imaging, 8:255-270, 2017. DOI 10.1007/s13244-017-0545-6

3. Jones, C. M. and Athanasiou, T., Is virtual bronchoscopy an efficient diagnostic tool for the thoracic surgeon? Ann Thorac Surg, 79:365-374, 2005. https://doi.org/10.1016/j.athoracsur.2004.0. 013.

4. Mirka, H., Ferda, J., Baxa, J., Multidetector computed tomography of chest trauma: indications, technique and interpretation. Insights Imaging, 3(5):433449, 2012. doi:10.1007/s13244-012-0187-7

5. Kotlyarov, P. M., Virtual Bronchoscopy for Tumors and Traumatic Lesions of the Airways [Online First], IntechOpen, 2019. DOI: 10.5772/intechopen.84562.

6. Matute, J. A., Gordillo, I., Garcia-Casillas, M. A., Romero, R., Lafuente, J., Vazquez, J., Fiberoptic bronchoscopy, 3-D reconstruction of the airway, and virtual bronchoscopy in patients with airway malformations. Preliminary report. Cir Pediatr, 16:116-120, 2003.

7. Schoepf, U. J., Seemann, M., Schuhmann, D., Bruning, R. D., Becker, C., Schwaiblmair, M., Müller, C., Knez, A., Haubner, M., Krapichler, C., Gebicke, K., Vogelmeier, C., Haberl, R., Englmeier, K. H., Reiser, M. F., Virtual, and threedimensional bronchoscopy with spiral, and electron beam computed tomography. Radiologe, 38:816-823, 1998. DOI: $10.1007 / \mathrm{s} 001170050429$

8. Maniatis, P. N., Triantopoulou, C. C., Tsalafoutas, I. A., Lamprakis, C. K., 
Malagari, K. S., Konstantinou, K., Christodoulou, E., Papailiou, J., Kelekis, D. A., Virtual Bronchoscopy versus Thin Section Computed Tomography in Evaluation of Moderate and Low Grade Stenoses: Receiver Operating Characteristic Curve Analysis. Acta Radiologica, 47(1):48-57, 2006. https://doi.org/10.1080/02841850500406837

9. Summers, R. M., Aggarwal, N. R., Sneller, M. C., Cowan, M. J., Wood, B.J., Langford, C.A., Wood, B. J., Langford, C. A., Shelhamer, J., H., CT virtual bronchoscopy of the central airways in patients with Wegener's granulomatosis. Chest 2002;121:242-50. https://doi.org/10.1378/chest.121.1.242

10.Maniatis, P., Triantopoulou, C., Tsalafoutas, I., Malagari, K., Siafas, I., Psatha, E., Koulentianos, E. D., Kelekis, D. A., Threshold selection in virtual bronchoscopy: phantom study and clinical implications. Acta Radiol, 45:176-83, 2004. DOI: $10.1080 / 02841850410003761$

11.Naidich, D. P., Lee, J. J., Garay, S. M., McCauley, D. I., Aranda, C. P., Boyd, A. D., Comparison of $\mathrm{CT}$ and fiberoptic bronchoscopy in the evaluation of bronchial disease. Am J Roentgenol, 148:1-7, 1987.
12.Hanley, J. A., McNeil, B. J., A method of comparing the areas under receiver operating characteristics curves derived from the same cases. Radiology,148:83943, 1983.

13.Anthracopoulosa, M. B., Alexopoulouc, E., Kagadis, G. S., Virtual Bronchoscopy and Other Three-Dimensional Imaging Methods. In: Priftis. K. N., Anthracopoulos, M. B., Eber, E., Koumbourlis, A.C., Wood, R.E. (eds): Paediatric Bronchoscopy. Prog Respir Res. Basel, Karger, 38, pp 95-112, 2010.

14.Lobo, L. and Antunes, D., Chest CT in infants and children. European Journal of Radiology, 82(7):1108-1117, 2013. https://doi.org/10.1016/j.ejrad.2011.12.006

15.Mitev, M. A., Trajkova, N., Arabadzhiev, D., Valkanov, S., Georgieva, N., Obretenov, E., Diagnostic capabilities of the virtual bronchoscopy at advanced neoplastic process of esophagus with formation of tracheobronchial fistula: description of a case. Trakia Journal of Sciences, $\quad$ 15(3):258-261, 2017. doi:10.15547/tjs.2017.03.015 